\title{
APPROXIMATION OF MULTIPLIERS
}

\author{
GARTH I. GAUDRY AND IAN R. INGLIS
}

\begin{abstract}
We note some necessary and sufficient conditions concerning norm approximation of Fourier multipliers, and give an example to show that $M_{q}(Z)$, the space of Fourier multipliers of type $(q, q)$, is not norm dense in $M_{p}(Z)$ when $1 \leqq q<p \leqq 2$. An extension of this example to more general groups is indicated.
\end{abstract}

1. Let $G$ and $\Gamma$ be dual L.C.A. groups with Haar measures $d x$ and $d \gamma$ respectively. Let $L^{p}(G), 1 \leqq p \leqq \infty$, be the usual Lebesgue space on $G$, $C_{0}(G)$ the space of continuous functions on $G$ which vanish at infinity, $C_{c}(G)$ the space of continuous functions on $G$ with compact support. The Fourier transform of a function $f$ is denoted $\hat{f}$. Let $M_{p}(\Gamma)$ denote the space of (equivalence classes of) essentially bounded measurable functions $\phi$ on $\Gamma$ which satisfy the condition

$$
\left|\int_{\Gamma} \phi(\gamma) \hat{f}(\gamma) \hat{g}(\gamma) d \gamma\right| \leqq C\|f\|_{p}\|g\|_{p^{\prime}}
$$

for any $f, g$ elements of $C_{c}(G)$ where $1 \leqq p \leqq \infty$ and $1 / p+1 / p^{\prime}=1$. The norm of $\phi$ is the least admissible value of $C$ in (1.1). It is known that

$$
L^{\infty}(\Gamma)=M_{2}(\Gamma) \supseteq M_{p}(\Gamma) \supseteq M_{q}(\Gamma) \supseteq M_{1}(\Gamma)=B(\Gamma)
$$

when $1 \leqq q \leqq p \leqq 2 ; B(\Gamma)$ is the space of Fourier transforms of complex regular Borel measures on $G$, with the inherited norm.

We shall make use of the fact that $M_{p}(\Gamma)$ is the dual of the Banach space $A_{p}(G)$ [4]. The space $A_{p}(G)$ consists of those functions in $C_{0}(G)$ which can be written in the form

$$
h=\sum f_{i} * g_{i}
$$

where $f_{i}, g_{i} \in C_{c}(G)$ and

$$
\sum\left\|f_{i}\right\|_{p}\left\|g_{i}\right\|_{p^{\prime}}<\infty
$$

Received by the editors April 30, 1973 and, in revised form, August 31, 1973.

AMS (MOS) subject classifications (1970). Primary 42A18; Secondary 42A68, 43A15, $41 \mathrm{~A} 35$.

Key words and phrases. Locally compact abelian group, multiplier, approximation. 
The norm of $h$ is the infimum of all sums (1.2') over all possible representations (1.2) of $h$. The duality between $A_{p}$ and $M_{p}$ is expressed by the formula

$$
\langle\phi, h\rangle=\sum \int_{\Gamma} \phi(\gamma) \hat{f}_{i}(\gamma) \hat{g}_{i}(\gamma) d \gamma
$$

where $\phi \in M_{p}(\Gamma)$ and $h \in A_{p}(G)$ with representation (1.2). One can readily deduce from the inclusion results among the multiplier spaces that

$$
C_{0}(G)=A_{1}(G) \supseteq A_{q}(G) \supseteq A_{p}(G) \supseteq A_{2}(G)=A(G)
$$

when $p$ and $q$ are as above and $A(G)$ is the space of Fourier transforms of functions in $L^{1}(\Gamma)$, with the inherited norm. Recall that $A(G)$ is dense in $A_{p}(G)$.

Our first result is an extension and variant of a result of Edwards [3] and Ramirez [6].

Proposition. Let $1 \leqq q<p \leqq 2$ and let $\phi$ be an element of $M_{p}(\Gamma)$. Then the following statements are equivalent:

(i) $\phi$ is approximable in the $M_{p}$ norm by elements of $M_{q}$.

(ii) If $\left(h_{n}\right)$ is a sequence in $A_{p}$ such that $\left\|h_{n}\right\| \leqq N$ and $\left\|h_{n}\right\|_{A_{q}} \rightarrow 0$, then $\left\langle\phi, h_{n}\right\rangle \rightarrow 0$.

(iii) If $\left(h_{\alpha}\right)$ is a net in $A_{p}$ such that $\left\|h_{\alpha}\right\| \leqq N$ and $\left\langle\psi, h_{\alpha}\right\rangle \rightarrow 0$ for each $\psi$ in $M_{q}$, then $\left\langle\phi, h_{\alpha}\right\rangle \rightarrow 0$.

Proof. It is simple to check that (i) implies (iii). As to the reverse implication, an application of the Hahn-Banach theorem shows that $\phi \in$ $M_{q}^{-}$if and only if every element $\lambda$ of $M_{p}^{*}$, of norm 1 , which annihilates $M_{q}$ also annihilates $\phi$. It follows from a well-known characterization of the unit ball in $M_{p}^{*}=A_{p}^{* *}$ that such a $\lambda$ is in the weak*-closure of the unit ball $U_{1}$ of $A_{p}$ (injected canonically into $M_{p}^{*}$ )-see [1, V.4.5]. With these observations in mind one concludes readily that (iii) implies (i).

Let $X$ denote the normed linear space consisting of the elements of $A_{p}(G)$ with $\|\cdot\|_{X}=\|\cdot\|_{A_{q}}$. Then (ii) simply means that the restriction of $\phi$ to the sets $U_{N}=\left\{h \in X:\|h\|_{A_{p}} \leqq N\right\}$ is (norm) continuous and (iii) means that the restriction of $\phi$ to the sets $U_{N}$ is weakly continuous. Clearly (iii) implies (ii). To prove the converse, suppose there exists a net $\left(h_{\alpha}\right)$ such that $\left\|h_{\alpha}\right\|_{A_{\nu}} \leqq N, h_{\alpha} \rightarrow 0$ weakly, and $\left\langle\phi, h_{\alpha}\right\rangle+0$. Then there exists a subnet which we shall also call $\left(h_{\alpha}\right)$ so that $\left\langle\phi, h_{\alpha}\right\rangle \rightarrow a \neq 0$. There exists an $\alpha_{0}$ so that if $\varepsilon=|a| / 2$, then $\left\langle\phi, h_{\alpha}\right\rangle \in B_{\varepsilon}(a)=\{z \in C:|z-a|<\varepsilon\}$, if $\alpha>\alpha_{0}$. Consider the set $S=\left\{h_{\alpha}\right\}_{\alpha>\alpha_{0}}$. 0 is certainly in the weak closure of $S$. It follows from Theorem V.3.13 of [1] that 0 belongs to the norm closure of co $S$, where co $S$ denotes the convex hull of $S$. So there exists a sequence $\left(y_{n}\right)$ in co $S$ so that $\left\|y_{n}\right\|_{A_{a} \rightarrow 0}$. By condition (iii), $\left\langle\phi, y_{n}\right\rangle \rightarrow 0$, but $y_{n} \in \operatorname{co} S$ so $\left\langle\phi, y_{n}\right\rangle \in$ co $B_{\varepsilon}(a)=B_{\varepsilon}(a)$. Hence $\left|\left\langle\phi, y_{n}\right\rangle\right|>a / 2$ and $\left\langle\phi, y_{n}\right\rangle \rightarrow 0$, a contradiction. 
COROLlary. Let $\phi$ be a continuous bounded function on $\Gamma$. Then the following statements are equivalent:

(i) $\phi$ is the uniform limit of Fourier-Stieltjes transforms.

(ii) If $\left(h_{n}\right)$ is a sequence in $A(G)$ such that $\left\|h_{n}\right\|_{A} \leqq N$ and $\left\|h_{n}\right\|_{\infty} \rightarrow 0$ then $\int_{\Gamma} \hat{h}_{n}(\gamma) \phi(\gamma) d \gamma \rightarrow 0$.

(iii) If $\left(h_{\alpha}\right)$ is a net in $A(G)$ such that $\left\|h_{\alpha}\right\|_{A} \leqq N$ and $\int_{\Gamma} \hat{h}_{\alpha}(\gamma) \psi(\gamma) d \gamma \rightarrow 0$ for each $\psi$ in $B(\Gamma)$ then $\int_{\Gamma} \hat{h}_{\alpha}(\gamma) \phi(\gamma) d \gamma \rightarrow 0$.

Remark. The equivalence of conditions (i) and (iii) of this Corollary is the result of Edwards and Ramirez [loc. cit.].

2. An explicit example. Figà-Talamanca and Gaudry [5] have constructed an element $\phi$ of $M_{p} \cap C_{0}(Z)$ which is not the limit in $M_{p}$ norm of elements of $A(Z)$. We show that the function $\phi$ constructed by them is not approximable in $M_{p}$ norm by elements of $M_{q}(Z)$ when $1 \leqq q<p \leqq 2$. By condition (ii) of the above Proposition it suffices to produce a sequence $\left(h_{n}\right)$ in $A_{p}(T)$ such that $\left\|h_{n}\right\|_{A_{p}} \leqq N$ for some $N,\left\|h_{n}\right\|_{A_{q}} \rightarrow 0$, and $\left\langle\phi, h_{n}\right\rangle \rightarrow 0$. Define the sequences $\left(\rho_{n}\right)$ and $\left(\sigma_{n}\right)$ of Rudin-Shapiro polynomials on $T$ as follows:

Then

$$
\begin{aligned}
\rho_{0} & =\sigma_{0}=1, \\
\rho_{n}(x) & =\rho_{n-1}(x)+\exp \left(i 2^{n-1} x\right) \sigma_{n-1}(x), \\
\sigma_{n}(x) & =\rho_{n-1}(x)-\exp \left(i 2^{n-1} x\right) \sigma_{n-1}(x) .
\end{aligned}
$$

$$
\left|\rho_{n}\right|^{2}+\left|\sigma_{n}\right|^{2}=2\left(\left|\rho_{n-1}\right|^{2}+\left|\sigma_{n-1}\right|^{2}\right)=\cdots=2^{n+1}
$$

from which it follows that $\left\|\rho_{n}\right\|_{\infty} \leqq 2^{(n+1) / 2}$. Further, the functions $\hat{\rho}_{n}$ and $\hat{\sigma}_{n}$ take only the values 0 and \pm 1 and are supported precisely on the set $\left\{0, \cdots, 2^{n}-1\right\}$.

On $\left[2^{n}, 2^{n+1}\right)$ put $\phi=\left(\exp \left(i 2^{n} x\right) \rho_{n}(x)\right)^{\wedge} \cdot 2^{-n / r}$ where $r=2 p /(2-p)$ and $n \geqq 0$. Put $\phi(k)=0$ for $k=0,-1,-2, \cdots$. Then $\phi \in M_{p} \cap C_{0}(Z)$-see [5]. Now $\rho_{n}=\rho_{n} * D_{2^{n}}$ where $D_{N}$ denotes the $N$ th Dirichlet kernel. It is known [2, Exercise 7.5] that $\left\|D_{N}\right\|_{p} \sim B_{p} N^{1 / p^{\prime}}$ as $N \rightarrow \infty$, when $1<p<\infty$ and $B_{p}$ is a constant depending only on $p$. Hence

$$
\left\|\rho_{n}\right\|_{A_{D}} \leqq\left\|\rho_{n}\right\|_{p^{\prime}}\left\|D_{2^{n}}\right\|_{p} \leqq B_{p^{2}} 2^{(n+1) / 2} 2^{n / p^{\prime}} \text { as } n \rightarrow \infty
$$

and, if $q>1$,

$$
\left\|\rho_{n}\right\|_{A_{q}} \leqq B_{q} 2^{(n+1) / 2} 2^{n / q^{\prime}} \text { as } n \rightarrow \infty .
$$

Put $h_{n}(x)=\exp \left(i 2^{n} x\right) \rho_{n}(x) / 2^{(n+1) / 2} 2^{n / p^{\prime}}$. Then $\left\|h_{n}\right\|_{A_{p}} \leqq B_{p}$ and $\left\|h_{n}\right\|_{A_{q}} \leqq$ $B_{q} 2^{n\left(1 / q^{\prime}-1 / p^{\prime}\right)}$ as $n \rightarrow \infty$. Since $1<q<p \leqq 2$ we have $2 \leqq p^{\prime}<q^{\prime}<\infty$ whence $\left\|h_{n}\right\|_{A_{a}} \rightarrow 0$ as $n \rightarrow \infty$. Finally

$$
\begin{aligned}
\left\langle\phi, h_{n}\right\rangle & =\rho_{n} * \rho_{n}(0) / 2^{(n+1) / 2} 2^{n / \alpha^{\prime}} 2^{n / r} \\
& =2^{n} / 2^{(n+1) / 2} 2^{n / p^{\prime}} 2^{n / r}=2^{-1 / 2}
\end{aligned}
$$


which completes the proof for $q>1$. But if $\phi \in M_{p} \mid M_{q}^{-}$for $1<q<p$, then certainly $\phi \notin M_{1}^{-}$, which completes the proof.

REMARKS. (a) It is possible to generalize the argument given above to the case of an arbitrary noncompact L.C.A. group $\Gamma$, and so to produce a function $\phi$ in $M_{p} \cap C_{0}(\Gamma) \backslash M_{a} \cap C(\Gamma)^{-}$. An indication of how to do this, by using the structure theorem and a lifting argument, is given in [5].

(b) It is possible to modify the above argument to prove that there exists $\phi \in M_{p} \cap C_{0}(Z)$ which is not the limit, in $M_{p}$ norm, of elements of $\bigcup_{1 \leqq a<p} M_{q}(Z)$. Take $\phi$ as above. Then it is easily seen that it suffices, given any sequence $\left(q_{n}\right)$ of real numbers $1 \leqq q_{n}<p$ such that $q_{n} \rightarrow p$, to produce a sequence $\left(h_{n}\right)$ in $A_{p}$ such that $\left\|h_{n}\right\|_{A_{p}} \leqq N,\left\|h_{n}\right\|_{A_{q_{n}}} \rightarrow 0$, and $\left\langle\phi, h_{n}\right\rangle \rightarrow 0$. Define the sequence $k(n)$ so that $k(n) \rightarrow \infty$ in such a way that $k(n)\left(1 / q_{n}-1 / p\right) \rightarrow \infty$, and put $h_{n}=\left\{\exp \left(i 2^{k(n)} x\right\} \rho_{k(n)} / 2^{(k(n)+1) / 2} 2^{k(n) / p^{\prime}}\right.$. It is easy to check that this yields the desired result.

(c) C. Fefferman (grapevine) is reported to have produced an element $\phi$ of $M_{n} \cap C_{c}\left(\boldsymbol{R}^{n}\right)$ such that $\left\|\phi_{a}-\phi\right\|_{M_{p}}+0$ as $a \rightarrow 0$, where $\phi_{a}(x)=\phi(x-a)$. An elementary interpolation argument shows that such a function $\phi$ is not approximable, in $M_{p}$ norm, by elements of $M_{q} \cap C\left(R^{n}\right)$.

\section{REFERENCES}

1. N. Dunford and J. T. Schwartz, Linear operators. I: General theory, Pure and Appl. Math., vol. 7, Interscience, New York, 1958. MR 22 \#8302.

2. R. E. Edwards, Fourier series: A modern introduction. Vol. I, Holt, Rinehart and Winston, New York, 1967. MR 35 \#7062.

3. - Uniform approximation on noncompact spaces, Trans. Amer. Math. Soc. 122 (1966), 249-276. MR 33 \#4659.

4. Alessandro Figà-Talamanca, Translation invariant operators in $L^{D}$, Duke Math. J. 32 (1965), 495-501. MR 31 \#6095.

5. A. Figà-Talamanca and Garth I. Gaudry, Multipliers of $L^{p}$ which vanish at infinity, J. Functional Analysis 7 (1971), 475-486. MR 43 \#2429.

6. Donald E. Ramirez, Uniform approximation by Fourier-Stieltjes transforms, Proc. Cambridge Philos. Soc. 64 (1968), 323-333. MR 36 \#4273.

School of Mathematical Sciences, The Flinders University of South Australia, Bedford Park, Australia 5042 